\title{
Rates and risk factors associated with hospitalization for pneumonia with ICU admission among adults
}

\author{
Aaron D. Storms ${ }^{1,2,10}$, Jufu Chen ${ }^{1}$, Lisa A. Jackson ${ }^{3}$, James D. Nordin ${ }^{4}$, Allison L. Naleway ${ }^{5}$, Jason M. Glanz ${ }^{6}$, \\ Steven J. Jacobsen ${ }^{7}$, Eric S. Weintraub ${ }^{8}$, Nicola P. Klein ${ }^{9}$, Paul M. Gargiullo ${ }^{1}$ and Alicia M. Fry ${ }^{1 *}$
}

\begin{abstract}
Background: Pneumonia poses a significant burden to the U.S. health-care system. However, there are few data focusing on severe pneumonia, particularly cases of pneumonia associated with specialized care in intensive care units (ICU).

Methods: We used administrative and electronic medical record data from six integrated health care systems to estimate rates of pneumonia hospitalizations with ICU admissions among adults during 2006 through 2010. Pneumonia hospitalization was defined as either a primary discharge diagnosis of pneumonia or a primary discharge diagnosis of sepsis or respiratory failure with a secondary diagnosis of pneumonia in administrative data. ICU admissions were collected from internal electronic medical records from each system. Comorbidities were identified by ICD-9-CM codes coded during the current pneumonia hospitalization, as well as during medical visits that occurred during the year prior to the date of admission.

Results: We identified 119,537 adult hospitalizations meeting our definition for pneumonia. Approximately 19\% of adult pneumonia hospitalizations had an ICU admission. The rate of pneumonia hospitalizations requiring ICU admission during the study period was 76 per 100,000 population/year; rates increased for each age-group with the highest rates among adults aged $\geq 85$ years. Having a co-morbidity approximately doubled the risk of ICU admission in all age-groups.
\end{abstract}

Conclusions: Our study indicates a significant burden of pneumonia hospitalizations with an ICU admission among adults in our cohort during 2006 through 2010, especially older age-groups and persons with underlying medical conditions. These findings reinforce current strategies aimed to prevent pneumonia among adults.

Keywords: Pneumonia/epidemiology, Intensive care units, Hospitalization, Trends, Rates, Adult

\section{Background}

Pneumonia poses a significant burden to the U.S. healthcare system. In 2010, pneumonia was one of the 10 leading causes of death in the U.S. [1] and resulted in over 50,000 deaths (a rate of 16.2 per 100,000 in the U.S. population) [2]. Approximately 1.1 million pneumonia associated hospital discharges (a rate of 366 per 100,000 U.S. population) have been reported [3]. However, there are few data focusing on severe pneumonia, such as

\footnotetext{
* Correspondence: afry@cdc.gov

${ }^{1}$ Influenza Division, Centers for Disease Control and Prevention, 1600 Clifton

Rd NE, Mailstop A32, Atlanta, GA 30333, USA

Full list of author information is available at the end of the article
}

cases of pneumonia requiring care in an intensive care unit (ICU), though these data could facilitate planning for resource utilization (i.e. use of hospital beds, ICU beds, and ventilators) during emergencies. We used administrative and electronic medical record data from six integrated health care systems to estimate rates of pneumonia hospitalizations with ICU admissions among adults during 2006 through 2010.

\section{Methods}

The Vaccine Safety Datalink (VSD) is a joint project between the Centers for Disease Control and Prevention (CDC) and eight integrated health care systems in the 
U.S. We used data from six of the eight VSD sites for this study (Group Health Cooperative [Washington], HealthPartners Institute [Minnesota], Kaiser Permanente of Colorado, Kaiser Permanente of Northern California, Kaiser Permanente Northwest [Colorado, California, Oregon], and Kaiser Permanente of Southern California), which comprises approximately 6 million enrollees per year $(2.5 \%$ of the 2010 U.S. population). Two sites were excluded as they did not consistently capture ICU admissions. Data collected from patients aged $\geq 18$ years enrolled in these sites include demographics (age, sex), dates of out-patient medical encounters or hospital admissions, dates of discharge, and International Classification of Diseases, Ninth Revision, Clinical Modification (ICD-9-CM) diagnosis and procedure codes for all out-patient and in-patient medical encounters [4].

Pneumonia hospitalization was defined by an expanded case definition [5] which included any of the following three possibilities: 1) primary discharge diagnosis of pneumonia (ICD-9-CM codes: 480-488); 2) primary discharge diagnosis of sepsis (ICD-9-CM codes: 038, 003.1, 020.2, 022.3, 036.2, 036.3, 054.5, 098.89, 785.52, 995.91, 995.92) with pneumonia listed as a secondary diagnosis in any diagnostic field; 3) primary discharge diagnosis for respiratory failure (ICD-9-CM codes: 518.81, 518.82, 518.84, 799.1) with pneumonia listed as a secondary diagnosis in any diagnostic field. Note that we added the influenza ICD-9-CM codes (487 and 488), which included seasonal and novel influenza viruses (i.e., H1N1pdm09), influenza pneumonia, as well as other influenza associated syndromes to the previously published "expanded pneumonia" codes as influenza can be associated with lower respiratory tract illness. Very few hospitalizations had influenza discharge codes (data not shown). We included persons aged $\geq 18$ years hospitalized during January 1, 2006 through December 31, 2010.

ICU admission was defined as an admission to any of the following hospital units during the pneumonia hospitalization: pediatric ICU, medical ICU, cardiac care unit, surgical ICU, neuro/neurosurgical ICU, transition or step-down unit. ICU admissions were identified by each of the sites using their internal electronic medical records and site-specific methods to query the electronic medical record for ICU admissions. We also assessed "assisted ventilation", which included invasive assisted ventilation and non-invasive interventions such as BiPAP and CPAP. We used assisted ventilation current procedure terminology (CPT) and ICD-9-CM procedure codes for assisted ventilation use (Additional file 1: Table S1). Monthly rates were calculated by dividing the monthly number of hospitalizations by the number of active enrollees during each month of the study period. Annual rates were calculated by dividing the number of hospitalizations each year by the yearly average of enrollees. 95\% confidence intervals (CI) were calculated around the rate estimates by using the Rothman-Greenland method [6]. Annual rates were age-group adjusted using the 2006 VSD population to standardize the proportion of each age-group for other years.

We assessed the association of age-group (18-49, 50$64,65-74,75-84, \geq 85$ years), sex, and comorbidities with ICU admission. Race and ethnicity data were not available. Comorbidities were identified by ICD-9-CM codes coded during medical visits that occurred during the year prior to the date of admission (i.e. in-patient and out-patient visits, including to the emergency department) as well as during the current pneumonia hospitalization. These included respiratory, cardiovascular, renal and liver diseases, diabetes, malignancies, immunosuppressive, hematological and neurological/musculoskeletal disorders (Additional file 2: Table S2). Crude odds-ratios (OR) were calculated for each comorbidity, in addition to male sex. Those factors that had a $P$ value $\leq 0.2$ were included in the multivariable logistic regression model. We assessed for effect modification due to age-group, and for interaction between diabetes, cardiovascular disease and renal disease. All analyses were conducted using SAS 9.3 (Cary, NC).

The Institutional Review Boards in each of the participating institutions reviewed and approved this study. No informed consent was required for this project.

\section{Results}

During January 1, 2006 through December 31, 2010, we identified 119,537 adult hospitalizations meeting our expanded case definition for pneumonia (Table 1). Most pneumonia hospitalizations were from the Northern California (36\%) and Southern California (42\%) regions of Kaiser Permanente while the remaining $22 \%$ were from the other four sites (range 4.3\% - 7.2\%). Of the included hospitalizations, only 87,457 (73\%) met the definition on the basis of a primary discharge diagnosis code of pneumonia, with the remainder meeting the definition on the basis of a primary discharge diagnosis of sepsis or respiratory failure and a secondary diagnosis of pneumonia (Additional file 3: Table S3). The rate of pneumonia hospitalization was 400 per 100,000 population/year for all adults. Among adults hospitalized for pneumonia, the median length of hospital stay was 4 days (interquartile range [IQR] 2-7 days). For patients hospitalized with pneumonia and admitted to the ICU, the median length of hospital stay was 6 days (IQR 4-12 days) with only minor variation by age-group (data not shown).

Approximately $19 \%$ of adult pneumonia hospitalizations had an ICU admission and 13\% had assisted ventilation (Table 1). Not all assisted ventilation was associated with an ICU admission code. Among 15,564 hospitalizations with assisted ventilation, 9557 (61\%) 


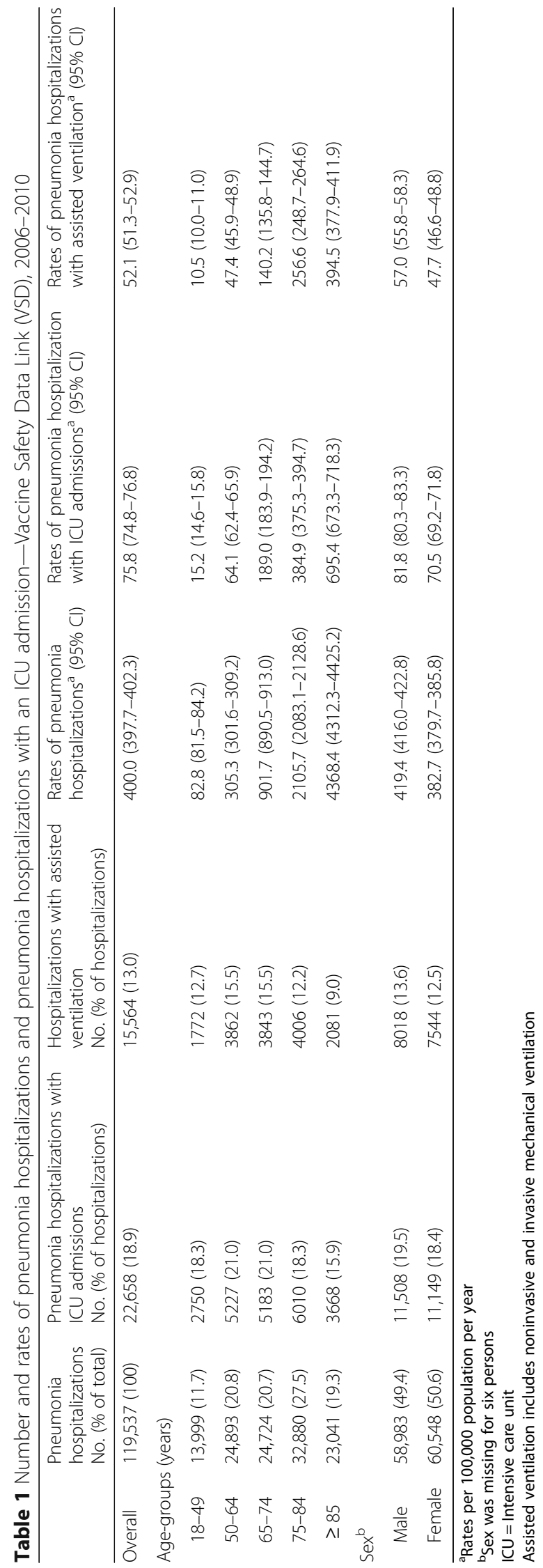


also had ICU admission. Overall, 42\% (9557/22,658) of pneumonia hospitalizations with ICU admission also had assisted ventilation. The highest proportion of ICU admissions occurred in the patients with a primary respiratory failure discharge code (Additional file 3: Table S3).

The rate of pneumonia hospitalization with ICU admission was 76 per 100,000 population/year for all adults (Table 1). The rates of pneumonia hospitalization, hospitalization with an ICU admission, and hospitalization with assisted ventilation increased for each age group with the lowest rates among adults aged 18-49 years. Rates for adults aged $\geq 85$ years were highest, with 53 times higher rates for pneumonia hospitalizations (4368 per 100,000), and 46 times higher rates for pneumonia hospitalizations with ICU admissions (695 per 100,000), compared to persons in the 18-49 age-group (83 per 100,000 for pneumonia hospitalizations, and 15 per 100,000 for hospitalizations with an ICU admission). Pneumonia hospitalization rates for all persons aged $\geq 65$ years were 1669 per 100,000 . The oldest age group ( $\geq 85$ years) had the lowest proportion of hospitalizations with an ICU admission and assisted ventilation. Rates were higher for males than for females for pneumonia hospitalizations (419 per 100,000 versus 383 per 100,000) and for hospitalizations with ICU admissions (82 per 100,000 versus 71 per 100,000) .

Annual rates of pneumonia hospitalization with ICU admissions ranged between 65.5 and 85.8 per 100,000. Rates during 2010 were higher than 2006. Rates were always higher in older age-groups, regardless of the year (Table 2).

The monthly rates of pneumonia hospitalization with ICU admission were consistently highest in the winter months during the 5-year study period, similar to pneumonia hospitalizations (Fig. 1). Seasonal variation was evident for all age groups (Additional file 4: Figure S1; Additional file 5: Figure S2).

Among hospitalized adults with pneumonia, the proportion with $>3$ co-morbidities was highest among the age groups $\geq 65$ years of age; approximately $50 \%$ of adults aged $65-74$ and $75-84$ had $>3$ co-morbidities. Having a co-morbidity approximately doubled the risk of ICU admission in all age-groups (Table 3). The risk of ICU admission did not change significantly with increasing number of comorbidities. Age was found to be an effect modifier, therefore; all analyses were stratified by age-group. On multivariable analysis, respiratory disease (excluding asthma) was an independent risk factor associated with ICU admission in all age-groups (Table 4). Malignancy was a risk factor for people aged $<75$ years, while male sex and immunosuppressive disorders were only risk factors for those aged $\geq 85$ years. Neurological/musculoskeletal disorders and liver disease were risk factors for some age-groups. Asthma was a protective factor for ICU admission in people aged $<75$ years. The risk of ICU admission associated with diabetes was not independent of the risk associated with cardiovascular disease. However, after accounting for them in the multivariable model, both comorbidities were still risk factors for ICU admission in all age-groups, and for most age-groups in people who presented both comorbidities.

\section{Discussion}

During 2006 through 2010, approximately 19\% of hospitalizations with pneumonia had an ICU admission among adults enrolled in 6 managed care organizations. We found rates of 400 and 76 per 100,000 population/year for pneumonia hospitalizations and pneumonia hospitalizations with ICU admission, respectively. The highest burden of pneumonia hospitalizations and hospitalizations with ICU admissions was among persons aged $\geq 85$ years; this group had rates 53 times higher for pneumonia and influenza hospitalizations, and 46 times higher for ICU admissions, compared to persons in the 18-49 age-group. Having a co-morbid condition approximately doubled the risk of an ICU admission associated with a pneumonia hospitalization.

Studies evaluating the incidence of severe pneumonia for adults are limited and the age-group specific rates for pneumonia hospitalization with ICU admission and assisted ventilation that we report are unique. Some studies have used ICD-9-CM codes to identify critical respiratory illness [7]; however, data on ICU admission are not reported in most administrative data sets. In addition, VSD data are unique in that they capture data from all enrolled adult members. Thus, unlike other databases, we were not limited to older adults with Medicare coverage or to datasets with all adults but with a subset of older adults with only certain types of Medicare insurance.

We found a similar proportion of pneumonia hospitalizations with ICU admission as reported by Jain et al. in a prospective study enrolling hospitalized patients with community acquired pneumonia (21\% and $19 \%)$ [8], but higher than other studies reporting that approximately $10 \%$ of patients hospitalized for community acquired pneumonia are admitted to the ICU [9-12]. This difference might be related to our pneumonia case definition that included admissions with primary diagnoses of sepsis or respiratory failure with pneumonia as secondary diagnosis. In our data, $12 \%$ of hospitalized patients with a primary diagnosis of pneumonia required an ICU admission, compared to $36 \%$ of those with sepsis and $46 \%$ of those with respiratory failure as the primary diagnoses, with pneumonia as a secondary diagnosis. Our decision to use this expanded definition of pneumonia is supported by recent literature suggesting that coding practices have changed in recent years, 
Table 2 Rates of pneumonia hospitalizations, pneumonia hospitalizations with an ICU admission, and pneumonia hospitalizations with assisted ventilation by year and age-group —Vaccine Safety Data Link (VSD), 2006-2010

\begin{tabular}{|c|c|c|c|c|c|c|}
\hline \multirow{2}{*}{$\begin{array}{l}\text { Years and age-groups } \\
2006\end{array}$} & \multicolumn{2}{|c|}{$\begin{array}{l}\text { Rates of pneumonia } \\
\text { hospitalizations }^{\mathrm{a}}(95 \% \mathrm{Cl})\end{array}$} & \multicolumn{2}{|c|}{$\begin{array}{l}\text { Rates of pneumonia hospitalization } \\
\text { with ICU admissions }{ }^{\mathrm{a}}(95 \% \mathrm{Cl})\end{array}$} & \multicolumn{2}{|c|}{$\begin{array}{l}\text { Rates of pneumonia hospitalizations } \\
\text { with assisted ventilation }{ }^{a}(95 \% \mathrm{CI})\end{array}$} \\
\hline & 376.7 & $(371.7-381.7)$ & 69.1 & $(67.0-71.2)$ & 47.9 & $(46.2-49.8)$ \\
\hline $18-49$ years & 67.3 & $(64.6-70.2)$ & 12.1 & $(11.0-13.4)$ & 8.6 & $(7.6-9.6)$ \\
\hline 50-64 years & 279.3 & (271.1-289.9) & 55.1 & $(51.5-57.6)$ & 42.5 & $(39.3-42.7)$ \\
\hline $65-74$ years & 886.5 & 861.4-912.4) & 182.5 & (171.3-194.4) & 136.4 & (126.8-146.8) \\
\hline $75-84$ years & 2122.6 & $(2071.8-2174.7)$ & 371.5 & $(350.6-393.7)$ & 252.9 & $(235.7-271.3)$ \\
\hline$\geq 85$ years & 4539.7 & $(4405.4-4678.1)$ & 721.8 & (669.4-778.3) & 388.1 & $(250.2-430.1)$ \\
\hline 2007 & 367.7 & $357.1-375.9)$ & 65.5 & $(61.2-70.2)$ & 46.2 & $(42.6-50.2)$ \\
\hline $18-49$ years & 70.3 & $(67.5-73.1)$ & 11.9 & $(10.8-13.1)$ & 9.2 & $(7.8-9.7)$ \\
\hline 50-64 years & 280.3 & $(272.2-278.5)$ & 58.6 & $(54.9-62.4)$ & 41.9 & $(43.5-50.1)$ \\
\hline $65-74$ years & 880.2 & (855.2-905.9) & 173.5 & $(162.6-185.1)$ & 126 & $(135.4-155.8)$ \\
\hline $75-84$ years & 2063.4 & (2013.1-2114.9) & 355.7 & (335.2-377.5) & 249.6 & $(239.4-275.2)$ \\
\hline$\geq 85$ years & 4085.7 & (3961.9-4213.3) & 554.2 & $(509.8-602.5)$ & 332.9 & (364.7-441.6) \\
\hline 2008 & 394.6 & (383.7-403.5) & 72.4 & $967.8-77.3)$ & 50.4 & $(46.6-54.5)$ \\
\hline $18-49$ years & 78.2 & $(75.2-81.2)$ & 13 & $(11.9-14.3)$ & 8.7 & (7.8-9.8) \\
\hline 50-64 years & 298.7 & $(290.5-298.6)$ & 61.5 & $(58.7-65.4)$ & 46.7 & $(43.5-50.1)$ \\
\hline $65-74$ years & 937.4 & (911.9-963/7) & 189.9 & $(178.6-202.0)$ & 145.2 & (135.4-155.8) \\
\hline $75-84$ years & 2164.6 & $(2113.3-2217.2)$ & 384.2 & $(362.9-406.7)$ & 256.7 & $(239.4-275.2)$ \\
\hline$\geq 85$ years & 4521.5 & $(4394.5-4652.2)$ & 707.1 & $(657.9-759.9)$ & 401.3 & (364.7-441.6) \\
\hline 2009 & 417.7 & $(406.5-429.1)$ & 85.8 & (80.8-91.0) & 57.3 & $(53.3-61.6)$ \\
\hline $18-49$ years & 112 & (108.4-15.6) & 22.7 & $(21.2-24.3)$ & 15.3 & $(14.0-16.7)$ \\
\hline 50-64 years & 358.3 & $(349.2-367.5)$ & 78.5 & $(74.3-82.9)$ & 56.3 & $(52.8-60.0)$ \\
\hline $65-74$ years & 928.8 & (903.9-954.3) & 207.5 & (195.9-219.7) & 150.2 & $(140.4-160.7)$ \\
\hline $75-84$ years & 2059.2 & (2009.7-2109.9) & 405.2 & $(383.5-428.0)$ & 262.3 & $(245.0-280.8)$ \\
\hline$\geq 85$ years & 4153.7 & $(4035.8-4275.1)$ & 744.6 & $(695.6-797.0)$ & 390.3 & $(355.2-428.7)$ \\
\hline 2010 & 394.32 & $(383.7-405.2)$ & 77.2 & $(72.6-82.1)$ & 52.8 & $(49.0-56.9)$ \\
\hline $18-49$ years & 86.7 & $(83.6-89.9)$ & 16.3 & $(15.0-17.8)$ & 10.7 & $(9.6-11.8)$ \\
\hline 50-64 years & 308 & (299.8-316.5) & 66.3 & $(62.5-70.3)$ & 49.1 & $(45.9-52.6)$ \\
\hline $65-74$ years & 876.1 & (852.6-900.3) & 190.3 & $(179.5-201.7)$ & 141.9 & (132.6-151.8) \\
\hline $75-84$ years & 2118.9 & (2069.3-2169.6) & 406.2 & (382.8-428.8) & 260.9 & $(243.9-279.1)$ \\
\hline$\geq 85$ years & 4537.3 & (4417.4-4660.4) & 736.6 & $(689.2-787.2)$ & 449.6 & $(412.9-589.5)$ \\
\hline
\end{tabular}

${ }^{a}$ Rates per 100,000 population per year. Yearly summary rates are age adjusted $\mathrm{ICU}=$ Intensive care unit

and that this expanded definition is better for identifying patients with pneumonia, in comparison to only using a primary discharge diagnosis of pneumonia, which may miss more severe cases $[5,13,14]$. Our finding of a lower proportion of ICU admissions in people aged $\geq 85$ years is in agreement with previously observed trends of a progressive decline in the proportion of pneumonia cases that are admitted to the ICU in older age-groups, though rates remain elevated compared with other age groups [15]. It is also consistent with an observed decrease in ICU utilization (all diagnoses) in older age-groups; more so in people aged $\geq 85$ years $[16,17]$. These trends may be related to increased hospitalization for pneumonia of lower severity with increasing age or less aggressive treatment among older individuals due to advance directives and living wills.

While several studies have reported rates for pneumonia hospitalizations, our rates may not be directly comparable to all published rates due to differences in age distributions and case definitions for pneumonia $[5,8,15,18,19]$. Our rates for pneumonia hospitalizations were similar to the adult age-group specific rates reported by Griffin et al. [20] during the 2007-2009 period. They used the National Inpatient Sample, a large all-payer nationwide inpatient 


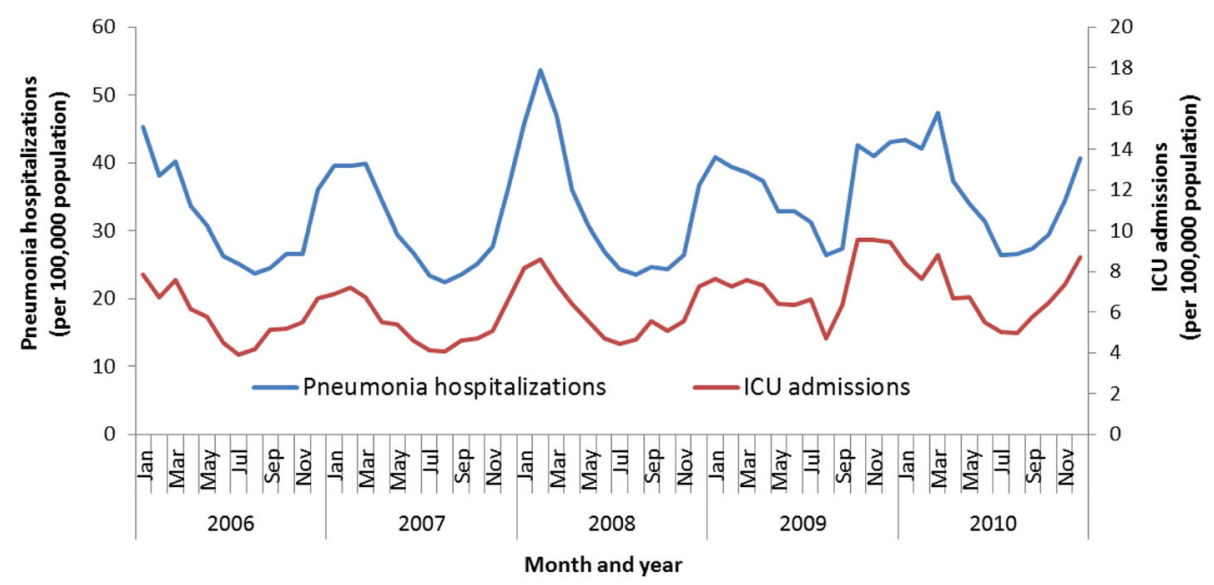

Fig. 1 Monthly rates of pneumonia hospitalizations and associated ICU admissions_Vaccine Safety Data Link (VSD), 2006-2010

Table 3 Number of comorbidities associated with ICU admission in patients hospitalized with pneumonia —Vaccine Safety Data Link (VSD), 2006-2010

\begin{tabular}{|c|c|c|c|c|}
\hline $\begin{array}{l}\text { Age-groups and number } \\
\text { of comorbidities }\end{array}$ & $\begin{array}{l}\text { Number in comorbidity strata } \\
\text { (\% within age group) }\end{array}$ & $\begin{array}{l}\text { No ICU admission } \\
(n=96,879) \\
\text { No. (row \%) }\end{array}$ & $\begin{array}{l}\text { ICU Admission } \\
(n=22,658) \\
\text { No. (row \%) }\end{array}$ & $\begin{array}{l}\text { Odds ratio } \\
(95 \% \mathrm{Cl})\end{array}$ \\
\hline $18-49$ years $(n=13,999)$ & & $11,429(81.6)$ & $2570(18.4)$ & \\
\hline 0 co-morbidities & $3687(26.3)$ & $3243(88.0)$ & $444(12.0)$ & REF \\
\hline 1 co-morbidity & $5630(40.2)$ & $4474(79.5)$ & $1156(20.5)$ & $1.89(1.68-2.12)$ \\
\hline 2 co-morbidities & $2205(15.8)$ & $1758(79.7)$ & $447(20.3)$ & $1.86(1.61-2.14)$ \\
\hline$\geq 3$ co-morbidities & $2477(17.7)$ & $1954(78.9)$ & $523(21.1)$ & $1.95(1.70-2.24)$ \\
\hline $50-64$ years $(n=24,893)$ & & $19,666(79.0)$ & $5227(21.0)$ & \\
\hline 0 co-morbidities & $2855(11.5)$ & $2517(88.2)$ & $338(11.8)$ & REF \\
\hline 1 co-morbidity & 8376 (33.6) & $6574(78.5)$ & $1802(21.5)$ & $2.04(1.80-2.31)$ \\
\hline 2 co-morbidities & $5272(2.21)$ & $4099(77.7)$ & $1173(22.3)$ & $2.13(1.87-2.43)$ \\
\hline$\geq 3$ co-morbidities & $8390(33.7)$ & $6476(77.2)$ & $1914(22.8)$ & $2.20(1.94-2.49)$ \\
\hline $65-74$ years $(n=24,724)$ & & $19,541(79.0)$ & $5183(21.0)$ & \\
\hline 0 co-morbidities & $1203(4.9)$ & $1076(89.4)$ & $127(10.6)$ & REF \\
\hline 1 co-morbidity & $5815(23.5)$ & $4639(79.8)$ & $1176(20.2)$ & $2.15(1.77-2.61)$ \\
\hline 2 co-morbidities & $5867(23.7)$ & $4628(78.9)$ & $1239(21.1)$ & $2.27(1.87-2.75)$ \\
\hline$\geq 3$ co-morbidities & $11,839(47.9)$ & $9198(77.7)$ & $2641(22.3)$ & $2.43(2.01-2.94)$ \\
\hline $75-84$ years $(n=32,880)$ & & $26,870(81.7)$ & $6010(18.3)$ & \\
\hline 0 co-morbidities & $1228(3.7)$ & 1100 (89.6) & $128(10.4)$ & REF \\
\hline 1 co-morbidity & $6765(20.6)$ & $5601(82.8)$ & $1164(17.2)$ & $1.79(1.47-2.17)$ \\
\hline 2 co-morbidities & $8363(25.4)$ & $6853(81.9)$ & $1510(18.1)$ & $1.89(1.56-2.29)$ \\
\hline$\geq 3$ co-morbidities & $16,524(50.3)$ & $13,316(80.6)$ & $3208(19.4)$ & $2.07(1.72-2.50)$ \\
\hline$\geq 85$ years $(n=23,041)$ & & $19,373(84.1)$ & $3668(15.9)$ & \\
\hline 0 co-morbidities & $1089(4.7)$ & 997 (91.6) & $92(8.4)$ & REF \\
\hline 1 co-morbidity & $5757(25,0)$ & $4865(84.5)$ & $892(15.5)$ & 1.99 (1.59-2.49) \\
\hline 2 co-morbidities & $6253(27.1)$ & $5256(84.1)$ & 997 (15.9) & $2.06(1.64-2.57)$ \\
\hline$\geq 3$ co-morbidities & $9942(43.1)$ & $8255(83.0)$ & $1687(17.0)$ & $2.21(1.78-2.76)$ \\
\hline
\end{tabular}


Table 4 Adjusted odds ratios for ICU admission for specific comorbidities in patients hospitalized with pneumonia, by age-group

\begin{tabular}{|c|c|c|c|c|c|}
\hline High risk condition & $\begin{array}{l}\text { Age 18-49 } \\
\text { Adjusted OR } \\
\text { (95\% Cl) }\end{array}$ & $\begin{array}{l}\text { Age 50-64 } \\
\text { Adjusted OR } \\
\text { (95\% Cl) }\end{array}$ & $\begin{array}{l}\text { Age 65-74 } \\
\text { Adjusted OR } \\
\text { (95\% Cl) }\end{array}$ & $\begin{array}{l}\text { Age } 75-84 \\
\text { Adjusted OR } \\
\text { (95\% Cl) }\end{array}$ & $\begin{array}{l}\text { Age } \geq 85 \\
\text { Adjusted OR } \\
(95 \% \text { Cl) }\end{array}$ \\
\hline Male sex & - & - & - & - & $1.11(1.03-1.19)$ \\
\hline Asthma & $0.85(0.76-0.95)$ & $0.81(0.75-0.88)$ & $0.88(0.81-0.96)$ & - & - \\
\hline Respiratory diseases (excluding asthma) & $1.33(1.20-1.47)$ & $1.12(1.05-1.20)$ & $1.10(1.03-1.17)$ & $1.14(1.05-1.18)$ & $1.09(1.01-1.18)$ \\
\hline Immunosuppressive disorders & - & - & - & - & $1.27(1.10-1.48)$ \\
\hline Malignancy & $1.21(1.05-1.40)$ & $1.16(1.07-1.25)$ & $1.15(1.07-1.23)$ & - & - \\
\hline Neurological/Musculosqueletal & $1.42(1.27-1.60)$ & $1.10(1.01-1.19)$ & - & $0.92(0.87-0.98)$ & - \\
\hline Liver disease & - & $1.16(1.02-1.32)$ & - & - & - \\
\hline \multicolumn{6}{|l|}{ Diabetes and cardiovascular disease } \\
\hline Diabetes without cardiovascular disease & $1.49(1.32-1.68)$ & $1.46(1.34-1.59)$ & $1.48(1.33-1.64)$ & $1.43(1.28-1.59)$ & $1.54(1.31-1.81)$ \\
\hline Cardiovascular disease without diabetes & $1.49(1.32-1.68)$ & $1.40(1.29-1.52)$ & $1.39(1.28-1.52)$ & $1.24(1.14-1.34)$ & $1.31(1.19-1.44)$ \\
\hline Diabetes and cardiovascular disease & - & $1.35(1.24-1.48)$ & $1.44(1.32-1.57)$ & $1.45(1.33-1.57)$ & $1.42(1.26-1.60)$ \\
\hline
\end{tabular}

"-"= Not included in final model, ICU = Intensive care unit

Hemoglobinopathies and renal disease dropped out of the final model in every age-group and therefore are not shown

care database, and defined pneumonia using ICD-9$\mathrm{CM}$ codes (primary discharge diagnosis of pneumonia, or primary diagnosis of meningitis, septicemia or empyema, with a secondary diagnosis of pneumonia). Jain et al. reported a hospitalization rate for radiographically confirmed pneumonia of 248 per 100,000 in a prospective study enrolling hospitalized patients with community acquired pneumonia [8]; this rate is lower than our estimate $(400$ per 100,000$)$ and may reflect the non-specific case definition for pneumonia that we used or issues related to defining radiologically confirmed pneumonia. Several studies have used administrative data to report rates of pneumonia hospitalizations $[5,15,18$, 19]. However, either the rates were higher and from earlier years [5], or the study used only first listed ICD-9-CM codes 480-486 for their definition of pneumonia [15, 18, 19]. Similar to our study, higher rates of pneumonia hospitalizations have been reported in older age-groups [15, 18 ] and older age is also associated with more serious outcomes [21, 22]. Our rates for pneumonia hospitalization for the $\geq 65$ age-group were comparable with other reports $[15,18]$.

In our cohort, patients with any comorbidity were more likely to have ICU admission. The presence of comorbidities has been reported to be an important determinant of the need for ICU admission in patients with community acquired pneumonia [9].

Overall, $13 \%$ of patients with a pneumonia hospitalization and $42 \%$ of patients with an ICU admission associated with pneumonia received assisted ventilation. Our findings are generally consistent with other reports although we captured assisted ventilation (noninvasive and invasive mechanical ventilation) rather than focusing on invasive mechanical ventilation. In Jain et al., $6 \%$ of adults hospitalized with radiographically confirmed community acquired pneumonia required invasive mechanical ventilation [8]. A recent study assessing a small cohort of pneumonia patients admitted to the ICU, reported that $38.6 \%$ received mechanical ventilation [23]. In another study of patients with community acquired pneumonia in Spain, $44 \%$ of those admitted to the ICU received mechanical ventilation [24]. Similar to the trends that we observed for ICU admissions, the proportion of admissions receiving assisted ventilation decreased in older age-groups, while the rate increased with increasing age. The upward trend in rates associated with older age has been identified in previous publications focusing on mechanical ventilation (all causes) $[25,26]$. The decreasing proportion of pneumonia hospitalizations that receive assisted ventilation among increasingly older adults is also similar to other reports [15].

There are several potential limitations to this study. We used discharge diagnosis codes to identify pneumonia hospitalizations, a standard but non-specific case definition for pneumonia. One consequence is our inability to differentiate between community-acquired and hospital-acquired pneumonia and the lack of radiology to confirm pneumonia. Although we present data from a large cohort collected from six sites throughout the U.S., most of the data are outcomes from two organizations in California (78\% of pneumonia hospitalizations). This might limit the generalizability of our findings to the general U.S. population. In addition, we did not validate the capture of ICU admissions; this was the first attempt to capture ICU admissions in this system and each site developed new programs to query the electronic medical record for ICU admissions. In addition, our estimates of assisted ventilation used procedure codes to identify assisted ventilation, and were not verified for completeness or accuracy with direct chart reviews. We were also 
unable to differentiate between invasive and non-invasive assisted ventilation (i.e. persons intubated and mechanically ventilated versus persons receiving support from BIPAP or CPAP) which is a distinction that carries clinical relevance.

\section{Conclusion}

In conclusion, we identified a significant burden of pneumonia hospitalizations requiring ICU admission during the study period. The highest rates for ICU admission were seen among older age-groups, and having underlying medical conditions was associated with ICU admissions. Obtaining ICU admission data from electronic medical records improved our ability to characterize pneumonia hospitalizations and our results could facilitate planning for resource utilization (i.e. use of hospital beds, ICU beds, and ventilators) during emergencies.

\section{Additional files}

Additional file 1: Table S1. Current Procedural Terminology (CPT) and ICD-9-CM Procedure Codes for Assisted ventilation, Vaccine Safety Data Link (VSD), 2006-2010. (DOCX 30 kb)

Additional file 2: Table S2. Current Procedural Terminology (CPT) and ICD-9-CM Procedure Codes for comorbidities, Vaccine Safety Data Link (VSD), 2006-2010. (DOCX 32 kb)

Additional file 3: Table S3. Number and proportion of hospitalizations with primary diagnosis of pneumonia, primary sepsis diagnosis with secondary pneumonia, or primary respiratory failure diagnosis with secondary pneumonia —Vaccine Safety Data Link (VSD), 2006-2010. (DOCX $41 \mathrm{~kb}$ )

Additional file 4: Figure S1A. Monthly rates of pneumonia with an ICU admission by age-group—Vaccine Safety Data Link (VSD), 2006-2010. (DOCX $90 \mathrm{~kb}$ )

Additional file 5: Figure S1B. Monthly rates of pneumonia with an ICU admission for age-groups 18-49 and 50-64. - Vaccine Safety Data Link (VSD), 2006-2010. (DOCX 76 kb)

\section{Abbreviations}

CDC: Centers for Disease Control and Prevention; Cl: confidence intervals; CPT: current procedure terminology; ICD-9-CM: Classification of Diseases, Ninth Revision, Clinical Modification; ICU: intensive care unit; IQR: interquartile range; VSD: vaccine safety datalink

\section{Acknowledgements}

We would like to acknowledge Mr. Brad Crane for his assistance with data management at KP Northwest.

\section{Funding}

The study was funded by the Centers for Disease Control and Prevention (CDC). CDC participated in the design of the study, analysis, interpretation of the data, and writing the manuscript.

\section{Availability of data and materials}

The data that support the findings of this study are available from the Vaccine Safety Datalink's (VSD) data sharing program with the Centers for Disease Control and Prevention's (CDC) National Center for Health Statistics (NCHS) Research Data Center, but restrictions apply to the availability of these data, which were used under license for the current study, and so are not publicly available. Data are however available from the authors upon reasonable request and with permission of VSD's data sharing program with the Centers for Disease Control and Prevention's NCHS Research Data Center.

\section{Disclaimer}

The findings and conclusions in this report are those of the authors and do not necessarily represent the official position of the Centers for Disease Control and Prevention.

\section{Authors' contributions}

Conception and design: AF, AS; Analysis and interpretation: AS, JC, PG; Drafting the manuscript for important intellectual content: AS, JC, LJ, JN, AN, JG, SJ, EW, NK, PG, AF. All authors have read and approved the final version of this manuscript.

\section{Ethics approval and consent to participate}

The Institutional Review Boards in each of the participating institutions (Group Health Cooperative, HealthPartners Research Foundation, Kaiser Permanente of Colorado, Kaiser Permanente of Northern California, Kaiser Permanente Northwest, and Kaiser Permanente of Southern California) reviewed and approved this study. No informed consent was required for this project.

\section{Consent for publication}

Not applicable.

\section{Competing interests}

The authors declare that they have no competing interests. Financial disclosures: Dr. Naleway has received research support from Medlmmune, Pfizer, and Merck for unrelated studies.

\section{Publisher's Note}

Springer Nature remains neutral with regard to jurisdictional claims in published maps and institutional affiliations.

\section{Author details}

${ }^{1}$ Influenza Division, Centers for Disease Control and Prevention, 1600 Clifton Rd NE, Mailstop A32, Atlanta, GA 30333, USA. "2Epidemic Intelligence Service, Centers for Disease Control and Prevention, Atlanta, USA. ${ }^{3}$ Group Health Cooperative, Seattle, USA. ${ }^{4}$ Health Partners Institute, Minneapolis, USA.

${ }^{5}$ Center for Health Research, Kaiser Permanente Northwest, Portland, USA.

${ }^{6}$ Kaiser Permanente of Colorado, Denver, USA. ${ }^{7}$ Kaiser Permanente of Southern California, Pasadena, USA. ${ }^{8}$ Immunization Safety Office, Centers for Disease Control and Prevention, Atlanta, USA. ${ }^{9}$ Kaiser Permanente of Northern California, Oakland, USA. ${ }^{10}$ Present Address: Keck School of Medicine of USC, 2020 Zonal Avenue, IRD 306, Los Angeles, CA 90033, USA.

Received: 18 July 2017 Accepted: 6 December 2017

Published online: 16 December 2017

\section{References}

1. Deaths, Percent of Total Deaths, and Death Rates for the 15 Leading Causes of Death: United States and Each State, 1999-2010. In.: National Vital Statistics System. 2012. Available at http://www.cdc.gov/nchs/nvss/ mortality/lcwk9.htm. Accessed 23 May 2013.

2. Deaths: Final Data for 2010. In.: National Vital Statistics Report (NVSR). Volume 61, Number 4. Available at https:/www.cdc.gov/nchs/data/nvsr/ nvsr61/nvsr61_04.pdf. Accessed 23 May 2013.

3. National Hospital Discharge Survey: 2010 table. In.: National Center for Health Statistics. Available at http://www.cdc.gov/nchs/data/nhds/2average/ 2010ave2_firstlist.pdf. Accessed 23 May 2013.

4. Baggs J, Gee J, Lewis E, Fowler G, Benson P, Lieu T, Naleway A, Klein NP, Baxter R, Belongia $\mathrm{E}$, et al. The vaccine safety datalink: a model for monitoring immunization safety. Pediatrics. 2011;127(Suppl 1):S45-53.

5. Lindenauer PK, Lagu T, Shieh MS, Pekow PS, Rothberg MB. Association of diagnostic coding with trends in hospitalizations and mortality of patients with pneumonia, 2003-2009. JAMA. 2012;307(13):1405-13.

6. Rothman KJ, Greenland S. Modern Epidemiology. Philadelphia: LippincottRaven Publishers; 1998.

7. Ortiz JR, Neuzil KM, Cooke CR, Neradilek MB, Goss CH, Shay DK. Influenza pneumonia surveillance among hospitalized adults may underestimate the burden of severe influenza disease. PLoS One. 2014;9(11):e113903.

8. Jain S, Self WH, Wunderink RG, Fakhran S, Balk R, Bramley AM, Reed C, Grijalva CG, Anderson EJ, Courtney DM, et al. Community-acquired pneumonia requiring hospitalization among U.S. adults. N Engl J Med. 2015; 373(5):415-27. 
9. Mandell LA, Wunderink RG, Anzueto A, Bartlett JG, Campbell GD, Dean NC Dowell SF, File TM Jr, Musher DM, Niederman MS, et al. Infectious Diseases Society of America/American Thoracic Society consensus guidelines on the management of community-acquired pneumonia in adults. Clin Infect Dis. 2007;44(Suppl 2):S27-72.

10. De Pascale G, Bello G, Tumbarello M, Antonelli M. Severe pneumonia in intensive care: cause, diagnosis, treatment and management: a review of the literature. Curr Opin Pulm Med. 2012;18(3):213-21.

11. Roson B, Carratala J, Dorca J, Casanova A, Manresa F, Gudiol F. Etiology, reasons for hospitalization, risk classes, and outcomes of communityacquired pneumonia in patients hospitalized on the basis of conventional admission criteria. Clin Infect Dis. 2001;33(2):158-65.

12. Luna CM, Palma I, Niederman MS, Membriani E, Giovini V, Wiemken TL, Peyrani P, Ramirez J. The impact of age and comorbidities on the mortality of patients of different age groups admitted with community-acquired pneumonia. Ann Am Thorac Soc. 2016;13(9):1519-26.

13. Ruhnke GW, Coca Perraillon M, Cutler DM. Mortality reduction among pneumonia patients still substantial despite the impact of coding changes. Am J Med. 2013;126(3):266-9.

14. Rothberg MB, Pekow PS, Priya A, Lindenauer PK. Variation in diagnostic coding of patients with pneumonia and its association with hospital riskstandardized mortality rates: a cross-sectional analysis. Ann Intern Med. 2014;160(6):380-8.

15. Kaplan V, Angus DC, Griffin MF, Clermont G, Scott Watson R, Linde-Zwirble WT. Hospitalized community-acquired pneumonia in the elderly: age- and sex-related patterns of care and outcome in the United States. Am J Respir Crit Care Med. 2002;165(6):766-72.

16. Angus DC, Barnato AE, Linde-Zwirble WT, Weissfeld LA, Watson RS, Rickert $T$, Rubenfeld GD. Robert Wood Johnson Foundation ICUE-O-LPG: use of intensive care at the end of life in the United States: an epidemiologic study. Crit Care Med. 2004;32(3):638-43.

17. Docherty A, Lone N, Anderson N, Walsh T. Epidemiology and outcomes of older patients admitted to Scottish intensive care units: a national database linkage study. Lancet. 2015;385(Suppl 1):S33.

18. Fry AM, Shay DK, Holman RC, Curns AT, Anderson LJ. Trends in hospitalizations for pneumonia among persons aged 65 years or older in the United States, 1988-2002. JAMA. 2005:294(21):2712-9.

19. Rate of discharges from short-stay hospitals, by age and first-listed diagnosis: United States, 2010. In.: National Center for Health Statistics. Available at http://www.cdc.gov/nchs/data/nhds/3firstlisted/2010first3_ rateage.pdf. Accessed 23 May 2013.

20. Griffin MR, Zhu Y, Moore MR, Whitney CG, Grijalva CG. U.S. hospitalizations for pneumonia after a decade of pneumococcal vaccination. N Engl J Med. 2013:369(2):155-63.

21. Fine MJ, Auble TE, Yealy DM, Hanusa BH, Weissfeld LA, Singer DE, Coley CM, Marrie TJ, Kapoor WN. A prediction rule to identify low-risk patients with community-acquired pneumonia. N Engl J Med. 1997;336(4):243-50.

22. Lim WS, van der Eerden MM, Laing R, Boersma WG, Karalus N, Town Gl, Lewis SA, Macfarlane JT. Defining community acquired pneumonia severity on presentation to hospital: an international derivation and validation study. Thorax. 2003:58(5):377-82.

23. Neamu R, Kerchberger V, Wise K, Kobaidze K, Leeper K. Re-evaluation of Severe Pneumonia Requiring ICU Admission: Results From a Prospective Observational Database. Chest. 2012; 142(4_MeetingAbstracts):231A

24. Cilloniz C, Ewig S, Ferrer M, Polverino E, Gabarrus A, Puig de la Bellacasa J, Mensa J, Torres A. Community-acquired polymicrobial pneumonia in the intensive care unit: aetiology and prognosis. Crit Care. 2011;15(5):R209.

25. Carson SS, Cox CE, Holmes GM, Howard A, Carey TS. The changing epidemiology of mechanical ventilation: a population-based study. J Intensive Care Med. 2006;21(3):173-82.

26. Wunsch $\mathrm{H}$, Linde-Zwirble WT, Angus DC, Hartman ME, Milbrandt EB, Kahn $J M$. The epidemiology of mechanical ventilation use in the United States. Crit Care Med. 2010:38(10):1947-53.

\section{Submit your next manuscript to BioMed Central and we will help you at every step:}

- We accept pre-submission inquiries

- Our selector tool helps you to find the most relevant journal

- We provide round the clock customer support

- Convenient online submission

- Thorough peer review

- Inclusion in PubMed and all major indexing services

- Maximum visibility for your research

Submit your manuscript at www.biomedcentral.com/submit

) Biomed Central 